\title{
Ring Shaped Cataract and Urinary Metabolites Among 2,4,6-trinitrotoulene Exposed Population of Pakistan
}

\section{Sidra Sarwat ( $\sim$ ssarwat137@gmail.com )}

UNSW: University of New South Wales https://orcid.org/0000-0002-4059-841X

\section{Ambreen Akhtar}

Al-Shifa Trust Eye Hospital Rawalpindi

Syeda Fizza Abdud Dayan

Al-Shifa Trust Eye Hospital Rawalpindi

Najma Shaheen

Al-Shifa Trust Eye Hospital Rawalpindi

Humna Shahid Durrani

Al-Shifa Trust Eye Hospital Rawalpindi

\section{Tariq Usman}

Al-Shifa Trust Eye Hospital Rawalpindi

Tayyab Afghani

Al-Shifa Trust Eye Hospital Rawalpindi

\section{Research Article}

Keywords: Ring-shaped cataract, TNT exposure, urinary metabolites of TNT and 4-ADNT

Posted Date: June 8th, 2021

DOl: https://doi.org/10.21203/rs.3.rs-526155/v1

License: (c) (i) This work is licensed under a Creative Commons Attribution 4.0 International License.

Read Full License 


\section{Abstract}

Background: Safety protocols are usually neglected among most of the TNT exposed population, therefore, rendering the community prone to various occupational hazards. The current study highlights ring shaped cataract and urinary metabolites of TNT among TNT exposed population $(n=26)$ against a control group $(n=20)$.

Methods: An observational case-control study was carried out in two groups: 300 subjects exposed to TNT in Dir and Bajour Agency, Pakistan and a control group from the base hospital. We determined the presence of ring shaped cataract and urine metabolites of TNT using slit lamp biomicroscope and gas chromatography mass spectrometric analysis respectively.

Results: Results substantiate a high level of urine metabolites for exposed subjects compared to the control group $(p<0.001)$. Age had no significant effect on $(p>0.05)$ on presence of ring shaped cataract and the level of urinary metabolites of TNT, while duration of exposure showed significant effect $(p<0.001)$. Females showed high incidence of ring shaped cataract and urinary metabolites of TNT than men $(p<0.001)$. The mean age of the exposed subjects was $51 \pm 14.38$ (Mean \pm SD) years. The mean year of exposure was $49 \pm 5($ Mean $\pm S D)$ years,

Conclusion: This study showed TNT as a risk factor for the presence of ring-shaped cataract among TNT exposed group in Pakistan. It is important to screen exposed community for the presence of ring-shaped cataract, and pre-clinical identification of TNT adducts to prevent systemic complications.

\section{Introduction}

2,4,6-trinitrotoluene (TNT) is an important occupational and environmental pollutant [1], has been employed as an explosive agent due to its relatively simple and economical production [2]. It was one of the main explosives used in World War 1 as well [3]. TNT exposure can induce well-documented systemic anomalies in humans such as hepatitis, aplastic anemia and cataract [4] The common route of TNT absorption is skin [5]. TNT can cause particular lenticular changes (ring-shaped cataract) even at low concentrations of environmental exposure [6,7] and the changes are potentially irreversible [4]. Ringshaped cataract is a brownish opacification in ocular lens caused by peroxidative denaturation of protein in lens fibre [8,9]. TNT initiates peroxidation through its cyclic reduction and oxidation by nitro reductases of mitochondria giving rise to superoxide anions. It is due to minimal antioxidant enzyme activity for defence against reactive oxygen species exogenously produced in the lens $[10,11]$. Ring-shaped cataract is a rare type of cataract that has been reported by few studies [12-15]. It was first observed in a 10 years old girl with a positive family history [16] and miners [12]. A ring-shaped cataract is categorized into four stages: the first stage appears as scattered whitish-grey opacity in the cortical layer on the nasal side, as a semicircular ring which may progress to a complete ring [17]. In the second stage, transparent spaces appear in circular opacities, causing them to appear strings of beads followed by $3^{\text {rd }}$ stage involving further opacification and centralization [17]. In the last stage, opacities increase in size and make the 
fundoscopy almost impossible to do [17]. A study showed that TNT is readily reduced to 4hydroxylamino2,6-dinitrotoluene (OH-4-ADNT), 4-amino-2,6-dinitrotoluene 4-ADNT) and 2-amino-4,6-dinitrotoluene (2ADNT) as intermediates that are further metabolized to 2,4-diamino-6-nitrotoluene (2,4-ADNT) and 2,6diamino-4-dinitrotoluene (2,6-ADNT) [18]. OH-4-ADNT was stable, therefore reduction of TNT to 4-ADNT is a two-step process. However, 2-ADNT could not be readily available in bloodstream or urine. Hence, the identification of 4-ADNT is a better biomarker for TNT [18]. As the literature indicates TNT as a causative agent for ring-shaped cataract $[19,20]$, therefore, it is imperative to evaluate the percentage of ringshaped cataract among TNT exposed population. TNT is extensively used by the local community under state law for mining purposes. In addition, this area was under war condition for the past 10 years. The study of ring-shaped cataract and its associated urine metabolites may provide substantial information about their prevalence and severity. It may help to formulate standardized preventive measures and create awareness about TNT related effects among practitioners and an exposed community population. This may identify the modifiable risk factors in preventing the problem under consideration [7]. Therefore, this study was aimed to compare the prevalence of TNT induced cataract and levels of urine metabolites in exposed and unexposed groups.

\section{Materials And Methods}

A case-control study was carried out in two groups: a 26 TNT exposed group from district Dir \& Bajour, Pakistan and a control group (20) with no TNT exposure from the base hospital. Detail history of the subjects was taken using a designed questionnaire. The extensive eye examination was done with the help of slit-lamp biomicroscope. Anterior segment photography was done with Carl-Zeiss Slit Lamp biomicroscope. All lens opacities matching Tiukina's description were classified as TNT cataract and graded on Tiukina's scale of stages 1-4. Subjects with systemic illnesses e.g. diabetes mellitus, hypertension, neurofibromatosis type 2 , atopic dermatitis, mentally challenged patients, pseudophakia and ocular pathology/trauma were excluded from the study. This study was approved by the ethics and scientific committee of Al-Shifa trust eye hospital, Rawalpindi, Pakistan. This study was done in consideration of Helniski protocol and data were collected with an informed consent form signed by subjects. Quantitative urinalysis for 4 ADNT was performed using gas chromatography-mass spectrometric analysis (GC-MS), Agilent technologies USA. $30 \mathrm{~mL}$ of spot urine was collected in plain sterilized commercial urine containers for urinalysis. Urine samples were stored at $-25^{\circ} \mathrm{C}$ prior to urinalysis. For assay of 4 ADNT certified reference material in water matrix was used for calibration of the equipment along with an internal standard of $\mathrm{N}$-Acetylaminobenzoic acid to validate the individual assay [21]. The data were analyzed using GraphPad (9.0.2). The results were shown as mean and standard deviation (SD) for quantitative variables, and frequency and percentage for qualitative variables. Twoway ANOVA was used for further analysis. A probability $(p)$ value less than 0.05 was taken as statistically significant.

\section{Results}


TNT and urinary metabolites of TNT, 4-amino-2,6-dinitrotoluene (4-ADNT) and 2-amino-4,6-dinitrotoluene (2-ADNT), were found among all exposed subjects. Besides, 2,6-dinitrotoluene (2,6 DNT) were detected among two-third of the exposed subjects and 2,4-dinitrotoluene (2,4 DNT) in one third. The levels of urine metabolites correlated strongly with the severity of ring-shaped cataract. The urine metabolites (4-ADNT AND 2-ADNT) were significantly associated with a risk of cataract. In the group of the exposed workers, $44 \%, 100 \%$ and $94 \%$ urine samples were positive for TNT, 4-ADNT and 2- ADNT respectively. $55.56 \%$ and $88.89 \%$ of urine samples were also tested positive for 2,6 DNT and 2,4 DNT. The levels of TNT, 4ADNT and 2-ADNT in urine were strongly associated with the stages of ring shaped cataract $(r=0.51$ 0.78). However, 2,4-DNT and 2,6-DNT do not correlate well $(r=0.47-0.55)$. As urine metabolites show the exposure within 48 hours, therefore, moderate correlation between urine levels and cataract stages was not unexpected.

\begin{tabular}{|llllll|}
\hline \multicolumn{7}{|c|}{ Table 1 } \\
\multicolumn{7}{|c|}{ Result of GC-MS among different stages of ring shaped cataract. } \\
\hline Tiukina's scale & TNT & 4-ADNT & 2-ADNT & 2,4-DNT & 2,6-DNT \\
\hline Stage 1 & 0.602 & 0.509 & 0.570 & 0.289 & 0.464 \\
\hline Stage 2 & 0.587 & 0.670 & 0.606 & 0.341 & 0.421 \\
\hline Stage 3 & 0.656 & 0.530 & 0.594 & 0.166 & 0.471 \\
\hline Stage 4 & 0.602 & 0.714 & 0.734 & 0.467 & 0.443 \\
\hline
\end{tabular}

The exposed group consisted of 19 female and 7 male (age $=51 \pm 14.4$ years, with $49+\underline{5}$ years of TNT exposure, while the unexposed group (control) consisted of 15 female and 5 male (age $=40 \pm 9$ years). Gender distribution showed a high proportion of females (88 \%) than men (12\%) in exposed subjects. According to the designed questionnaire, the occupational history of the exposed community population revealed a higher proportion of women working in the fields and rock blasting for the construction of houses. All cases in both groups were not suffered from diabetes mellitus, hypertension, atopic dermatitis, and ocular pathology/trauma.

Slit-lamp biomicroscopy showed specific TNT-induced ring shaped cataract among TNT exposed subjects. $1^{\text {st }}$ stage and $2^{\text {nd }}$ stages were $23.9 \%$ and $62.5 \%$ among exposed subjects with no significant effect on visual acuity $(p>0.05)$. However, $3^{\text {rd }}$ and $4^{\text {th }}$ stages were found only in $13.6 \%$ of the patients with a significant reduction in visual acuity in the $4^{\text {th }}$ stage. The ring-shaped cataract symmetrical, and the majority of cases were bilateral. However, seven cases were unilateral in the exposed community population. The control group showed lenticular changes, but a ring-shaped cataract was not observed in any subject. The ring-shaped cataract caused no effect on visual acuity in the first and second stage (Figure 1) and, the majority of subjects had the first stage of ring-shaped cataract. One case was reported as NPL with no fundal glow making it difficult to perform fundoscopy. Coloured anterior segment photography showed the central opacification of the lens in the shape of a ring as shown in figure 1. 
Figure $1 \mathrm{a}$ and $\mathrm{b}$ shows approximately 4-5 cm opacification in both eyes of the subject. However, the central part was clear and causing no effect on the visual axis. Similarly, the peripheral area around central ring-shaped cataract was clear and doesn't interrupt vision. Figure 1 shows further opacification of the lens, indicating the $3^{\text {rd }}$ state of the ring-shaped cataract. The opacification was denser, hence blocking the visual axis higher than $1^{\text {st }}$ stage. As shown in figure 1 , the number of patients with $1^{\text {st }}$ and $2^{\text {nd }}$ stage cataract was higher than the $3^{\text {rd }}$ and $4^{\text {th }}$ stage in exposed community subjects.

\section{Discussion}

In the present study, the ocular effects of TNT exposure using slit-lamp biomicroscopic examination and urine metabolites were investigated. TNT is considered a major occupational and environmental pollutants, therefore exposed groups are prone to various toxic complications [17]. This is the first study where ring shaped cataract was detected and associated urine metabolites of TNT were analysed in the non-captive TNT exposed community of Pakistan. Prevalence of cataract and urinary metabolites of TNT; 4-ADNT and 2-ADNT between exposed and unexposed subjects were significantly different. The presence of ring-shaped cataract along with positive urinary metabolites may predispose the possible systemic complications in the exposed population. Other studies have also shown higher prevalence among TNT exposed community population as well as factory workers [7, 11, 14, 22], which indicates the possibility of future occurrence in exposed regions of Pakistan. The mean age of the subjects diagnosed with ring-shaped cataract was comparable to the mean ages of other studies $[6,14,15]$ suggesting high chances of occurrence in the middle age group, which might be related to the duration of exposure. Gender distribution of ring-shaped cataract shows a high number of females and, it can be explained by social gender discrimination. The area under investigation was mountainous, therefore people use explosive's mining to build their houses. The reason may be their cultural and social norms, which involve extensive work by females outside their homes. Although gender difference was also found among the Danish arm factory workers, however, in contrast, no female was diagnosed with ring-shaped cataract [6]. The average years of exposure for the exposed population were higher than spirometry study of TNT workers [15] and Danish arm factory workers [6]. Although, this study supports the effect of exposure on the development of ring-shaped cataract, whereas no relation was found with age. This study showed reduced visual acuity at the $3^{\text {rd }}$ and $4^{\text {th }}$ stages of cataract. Another study showed no significant effect on visual acuity in subjects at any stage of the cataract [6] however, the study showed a significant reduction in visual acuity at the $4^{\text {th }}$ stage with no impact in the first three stages [14]. Data showed a higher proportion of subjects with a $2^{\text {nd }}$ grade of cataract as compared to other stages. There was only one case detected with stage 4 among exposed subjects. Still, a high percentage of the $2^{\text {nd }}$ and $3^{\text {rd }}$ stage may predispose progression to the $4^{\text {th }}$ stage, consequently leading to total blockage of the visual axis. The exposed group was not wearing any personal protective equipment (PPE). Therefore, the cases exposed to TNT without PPE longer period might have given convincing results.

The urine levels of TNT metabolites were similar to a study performed in a Chinese factory [23]. Similarly, a German study showed a level of 4ADNT $(0.23 \mathrm{mg} / \mathrm{mL}$ in 32 subjects) and $(0.71 \mathrm{mg} / \mathrm{mL}$ in 9 subjects) 
[6]. The levels of urine metabolites including 4-ADNT were related to the health status of the exposed population [19]. As we found similar results during urinalysis therefore, the same disease could appear in other population throughout year exposure. Our results are supported by a study that showed that TNT is readily reduced to 4hydroxylamino-2,6-dinitrotoluene (OH-4-ADNT), 4-amino-2,6-dinitrotoluene 4-ADNT) and 2-amino-4,6-dinitrotoluene (2-ADNT) as intermediates that are further metabolized to 2,4-diamino-6nitrotoluene (2,4-ADNT) and 2,6-diamino-4-dinitrotoluene (2,6-ADNT) [18]. This finding broadly supports the work of other studies in this area linking ring-shaped cataract with TNT. TNT can affect humans in several ways; however, this study could not measure all the possible effects due to the unavailability of multiple methodology protocols or instruments. This study was also limited by the absence of information about external dose, skin exposure and air measurement of TNT. Notwithstanding these limitations, the study suggests systemic investigations including $\mathrm{Hb}$ adducts of TNT for future studies. Further pathological assessment can signify the association of TNT with the occurrence of cataract and help to predict the severity of systemic side effects. This study has also resulted in an initiative of the standard clinical screening program to identity toxic manifestations of TNT and help reduce it impact on human health being.

\section{Conclusion}

We have found TNT as a chemical causing a meaningful increase in cataract prevalence in exposed group of Pakistan [4] This study has identified the rise in various urine metabolites of TNT among TNT exposed population in Pakistan. These findings may have significant implications for the understanding of risk assessment of people exposed to TNT.

\section{Declarations}

\section{Funding}

This project was funded by the Al-Shifa Trust Eye Hospital, Jhelum Road, Rawalpindi, Pakistan.

\section{Conflicts of interest/Competing interests}

There is no conflict of interest associated with this study.

\section{Availability of data and material}

Not applicable

\section{Code availability}

Not applicable 


\section{Ethics approval}

This study was approved by the ethics and scientific committee of Al-Shifa trust eye hospital, Rawalpindi, Pakistan.

\section{Consent to participate}

This study was done in consideration of Helniski protocol and data were collected with an informed consent form signed by subjects.

\section{Consent for publication}

Informed consent was taken for publication of the eye images.

\section{Acknowledgement}

We would like to acknowledge Armed Forces Institute of Pathology for help with systemic investigations.

\section{References}

1. Burrows, E.P., D.H. Rosenblatt, W.R. Mitchell, and D.L. Parmer, Organic Explosives and Related Compounds. Environmental and Health Considerations, R. Army Biomedical and M.D. Development Lab Fort Detrick, Editors. 1989

2. Yaws, C.L., Handbook of chemical compound data for process safety : comprehensive safety and health-related data for hydrocarbons and organic chemicals : selected data for inorganic chemicals. 1997, Houston, Tex.: Houston, Tex. : Gulf Pub. Co.

3. Gupta, R.C., Handbook of toxicology of chemical warfare agents. 2nd ed. ed. 2015: London, England : Elsevier : AP.

4. Naderi, M., M. Ghanei, M. Shohrati, A. Saburi, et al., Systemic complications of trinitrotoluene (TNT) in exposed workers. Cutan Ocul Toxicol, 2013. 32(1): p. 31-4.

5. Darrach, M.R., A. Chutjian, and G.A. Plett, Trace Explosives Signatures from World War II Unexploded Undersea Ordnance. Environmental Science \& Technology, 1998. 32(9): p. 1354-1358.

6. Kruse, A., M. Hertel, M. Hindsholm, and S. Viskum, Trinitrotoluene (TNT)-induced cataract in Danish arms factory workers. Acta Ophthalmol Scand, 2005. 83(1): p. 26-30.

7. Harkonen, H., M. Karki, A. Lahti, and H. Savolainen, Early Equatorial Cataracts in Workers Exposed to Trinitrotoluene. American Journal of Ophthalmology, 1983. 95(6): p. 807-810.

8. Hoenders, H.J. and H. Bloemendal, Lens Proteins and Aging. Journals of Gerontology, 1983. 38(3): p. 278-286. 
9. Krol, D.S. and V.P. Kolevatykh, [Ring-shaped cataract in chronic poisoning by trinitrotoluene (TNT)]. Oftalmol Zh, 1965. 20(3): p. 180-3.

10. Zitting, A., G. Szumanska, J. Nickels, and H. Savolainen, Acute Toxic Effects of Trinitrotoluene on RatBrain, Liver and Kidney - Role of Radical Production. Archives of Toxicology, 1982. 51(1): p. 53-64.

11. Savolainen, H., R. Tenhunen, and H. Härkönen, Reticulocyte haem synthesis in occupational exposure to trinitrotoluene. British Journal of Industrial Medicine, 1985. 42(5): p. 354-355.

12. Logan, I.M., Z.M. Skripnichenko, and E.T. Tkachenko, [Trinitrotoluene (TNT) cataract in miners, its diagnosis and prevention]. Oftalmol Zh, 1970. 25(8): p. 579-84.

13. Kamboj, A., H.A. Spiller, M.J. Casavant, T. Chounthirath, et al., Ocular Exposures Reported to United States Poison Control Centers. Ophthalmic Epidemiology, 2018: p. 1-11.

14. Lewis-Younger, C.R., N. Mamalis, M.J. Egger, D.O. Wallace, et al., Lens opacifications detected by slitlamp biomicroscopy are associated with exposure to organic nitrate explosives. Archives of Ophthalmology, 2000. 118(12): p. 1653-1659.

15. Shohrati, M., B. Najafian, A. Saburi, E. Vahedi, et al., Spirometric Findings in TNT Factory Workers Compared with Unexposed Controls. Asia Pacific Journal of Medical Toxicology, 2014. 3(3): p. 110114.

16. Haro, E., Hereditary disk-shaped (ring) cataract: Report on a family, with microscopic examination of an eye. Archives of Ophthalmology, 1946. 36(1): p. 82-100.

17. Yinon, J., Toxicity and Metabolism of Explosives. 1990: CRC Press.

18. Leung, K.H., M. Yao, R. Stearns, and S.-H.L. Chiu, Mechanism of bioactivation and covalent binding of 2,4,6-trinitrotoluene. Chemico-Biological Interactions, 1995. 97(1): p. 37-51.

19. Sabbioni, G., Y.Y. Liu, H. Yan, and O. Sepai, Hemoglobin adducts, urinary metabolites and health effects in 2,4,6-trinitrotoluene exposed workers. Carcinogenesis, 2005. 26(7): p. 1272-9.

20. Teir, H. and B. Grenquist-Nordén, Peripheral cataracts and trinitrotoluene exposure: A follow-up study. Acta Ophthalmologica, 1990. 68(195 S): p. 49-51.

21. Vorísek, V., M. Pour, K. Ubik, V. Hassmanová, et al., Analytical monitoring of trinitrotoluene metabolites in urine by GC-MS. Part I. Semiquantitative determination of 4-amino-2,6-dinitrotoluene in human urine. J Anal Toxicol, 2005. 29(1): p. 62-5.

22. Zhou, A.S., A clinical study of trinitrotoluene cataract. Polish journal of occupational medicine, 1990. 3(2): p. 171-176.

23. Sabbioni, G., J. Wei, and Y.Y. Liu, Determination of hemoglobin adducts in workers exposed to 2,4, 6trinitrotoluene. J Chromatogr B Biomed Appl, 1996. 682(2): p. 243-8.

\section{Figures}



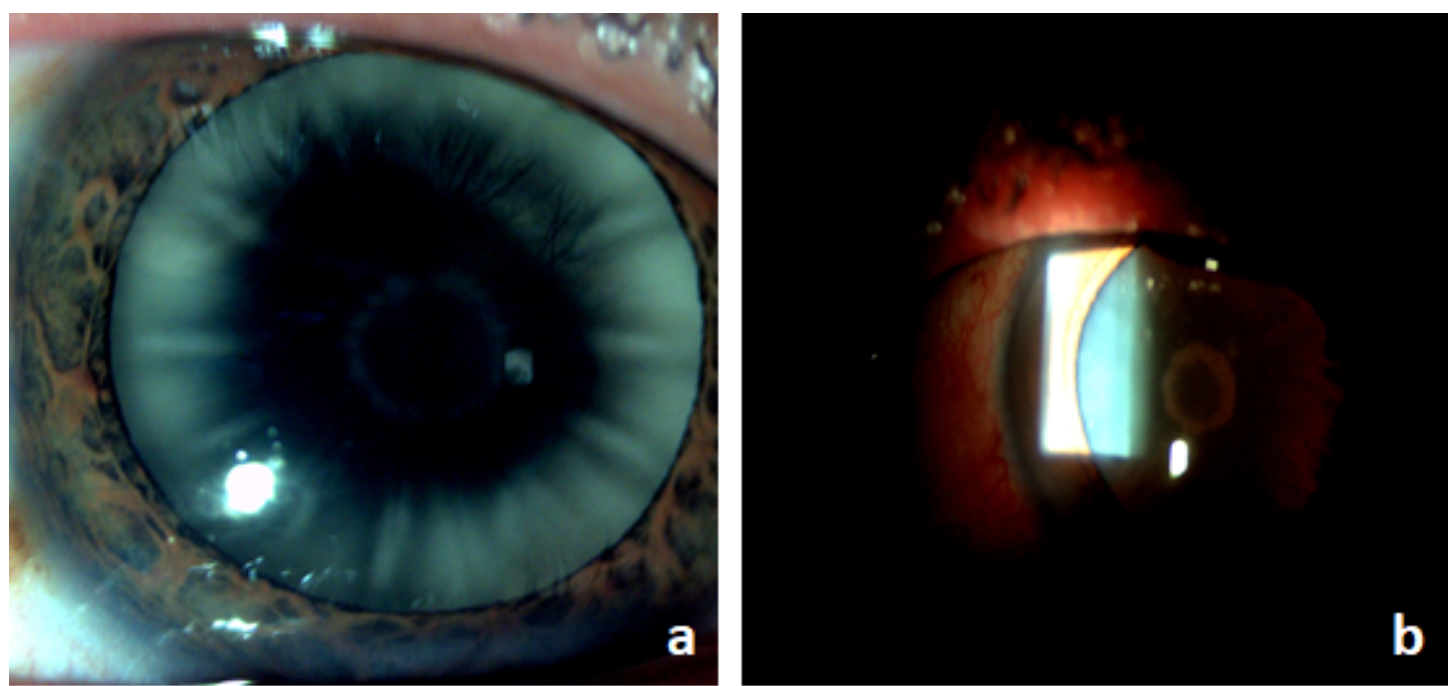

Figure 1

a \& b shows ring-shaped cataract respectively by anterior segment photography 